A\&A 521, E1 (2010)

DOI: $10.1051 / 0004-6361 / 201015613$

(C) ESO 2010

Herschel/HIFI: first science highlights Astrophysics

\title{
Editorial
}

\section{Herschel/HIFI: first science highlights}

The special feature which we publish in the current volume of A\&A is devoted to the first scientific data obtained with the HIFI (Heterodyne Instrument for the Far Infrared) instrument on board the Herschel satellite. Earlier this summer (vol. 518), we published a special feature devoted mainly to the SPIRE and PACS instruments on Herschel with cameras observing (mainly) continuum emission between 50 and 300 microns wavelength. HIFI provides complementary spectroscopic data (see de Graauw et al. 2010, A\&A, 518, L6) with exquisite spectral resolution. This provides unparalleled information on the kinematics of star forming regions as well as on hydrides in the diffuse ISM. We expect that it will serve as a pathfinder for future Herschel observations as well as for airborne observatories like SOFIA.

C. M. Walmsley, C. Bertout, F. Combes, T. Forveille, T. Guillot, A. Jones, and S. Shore Astronomy E Astrophysics Editors 